\title{
A Model for Electron Scattering in Irradiated Pyrochlore-Fluorite Systems
}

\author{
G.R. Lumpkin, ${ }^{*}$ M.G. Blackford,* K.L. Smith, ${ }^{*}$ K.R. Whittle, ${ }^{*}$ and N.J. Zaluzec** \\ *Australian Nuclear Science and Technology Organisation, PMB1, Menai, NSW 2234, Australia \\ **Argonne National Laboratory, 9700 South Cass Avenue, Argonne, IL 60439, USA
}

Certain minerals, materials for energy, and high technology devices may undergo irradiation, either internally through alpha decay, or externally by neutrons or ions [1]. Many of these materials become amorphous when irradiated beyond a critical dose level. Electron diffraction patterns are routinely used to study the structure of these materials, including the observation of order-disorder phenomena during irradiation [2]. Here we show how the intensities of Bragg beams change from a dynamical to a kinematic-like state during the accumulation of amorphous domains in the material.

The transition from the crystalline to the amorphous state can be considered as a two-phase problem, with $f_{\mathrm{c}}+f_{\mathrm{a}}=1$, where $f_{\mathrm{c}}$ and $f_{\mathrm{a}}$ are the fractions of the crystalline and amorphous phases, both having the same composition. For $f_{\mathrm{c}}=1$ the perfect crystal scatters into the nodes of the reciprocal lattice according to the many-beam dynamical equations and for $f_{\mathrm{a}}=1$ the amorphous phase scatters according to the Debye equation. Figure 1 shows the strong Bragg-Bragg interactions that occur in the perfect crystal according to relationships like $(\mathrm{hkl})_{1}+(\mathrm{hkl})_{2}=(\mathrm{hkl})_{3}$. However, once there are sufficient amorphous domains in the material, the diffuse scattering from these domains will begin to attenuate the double diffraction relationship between Bragg beams via relationships like $(\mathrm{hkl})_{1}+$ $(\mathrm{am})_{1}=(\mathrm{am})_{2}$ and $(\mathrm{am})_{1}+(\mathrm{hkl})_{1}=(\mathrm{am})_{2}$. Multiple diffuse scattering, e.g., $(\mathrm{am})_{1}+(\mathrm{am})_{2}=(\mathrm{am})_{3}$, will eventually come into play as shown in Figure 1. Thus, the relative intensities of the Bragg beams change dramatically with increasing radiation dose as $f_{\mathrm{a}}$ increases. The above model also suggests that the intensity scattered from the amorphous phase will be dependent upon the orientation of the specimen and that Bragg-diffuse interactions may be observed.

We tested this model by irradiating TEM samples of $\mathrm{Gd}_{2} \mathrm{Ti}_{2} \mathrm{O}_{7}$ in the IVEM-Tandem Facility at Argonne National Laboratory using 1.0 MeV Kr ions. The top row of Figure 2 reveals a systematic change in the Bragg beam intensities of the crystalline fraction from a dynamical pattern toward a kinematic-like pattern for the [110] zone axis with increasing dose. The weaker superlattice beams are lost in the amorphous background or in the enhanced small angle scattering. As shown previously [3], kinematically strong beams always the last to disappear during irradiation. Additional dynamical scattering effects are shown in the bottom row of Figure 2, in which the specimen is tilted to a $\langle 111\rangle$ systematic row. These SAED patterns exhibit stronger scattering at lower dose levels from the amorphous fraction due to the removal of numerous Bragg beams from the Ewald sphere upon tilting, together with multiple Bragg-diffuse scattering along the systematic row. Certain superlattice beams (e.g., 333) can be observed to high dose levels, indicating that the crystalline domains retain the ordered pyrochlore structure.

The model provides a general description of changes in Bragg beam intensities during the crystalline-amorphous transformation, but it is especially relevant to pyrochlore-fluorite systems in which the superlattice beams are very weak in the kinematic-like state. This has major implications for the interpretation of order-disorder phenomena for different damage mechanisms.

\section{References}

[1] Ewing, R.C., Weber, W.J., Lian, J., J. Appl. Phys. 95, 5949-5972 (2004).

[2] Lian, J., Chen, J., Wang, L.M., Ewing, R.C., Farmer, J.M., Boatner, L.A., Helean, K.B., Phys. Rev. B 68, 134107 (2003).

[3] Smith, K.L., Zaluzec, N.J., Lumpkin, G.R., In situ studies of ion irradiated zirconolite, pyrochlore, and perovskite. J. Nucl. Mater. 250, 36-52 (1997). 

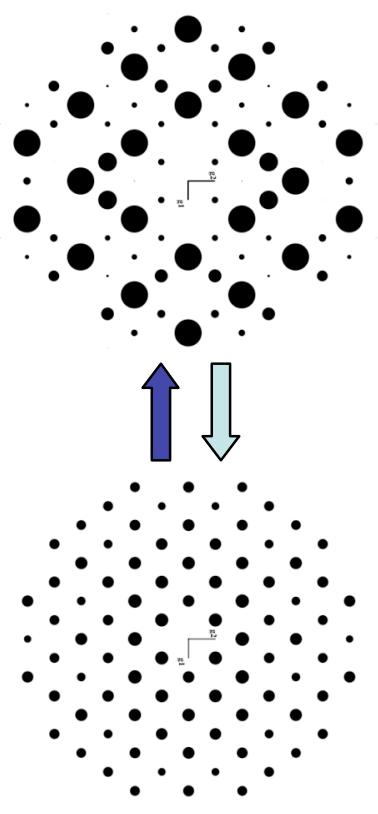

Fig. 1. Far left: Calculated [110] SAED patterns for for $\mathrm{Gd}_{2} \mathrm{Ti}_{2} \mathrm{O}_{7}$ under kinematical (top) and dynamical conditions (bottom, thickness $=50 \mathrm{~nm}$ ). Right: Model for electron scattering with increasing dose from left to right: build up of amorphous domains attenuates the Bragg-Bragg scattering, leading to a transition from a dynamical to kinematic-like state.
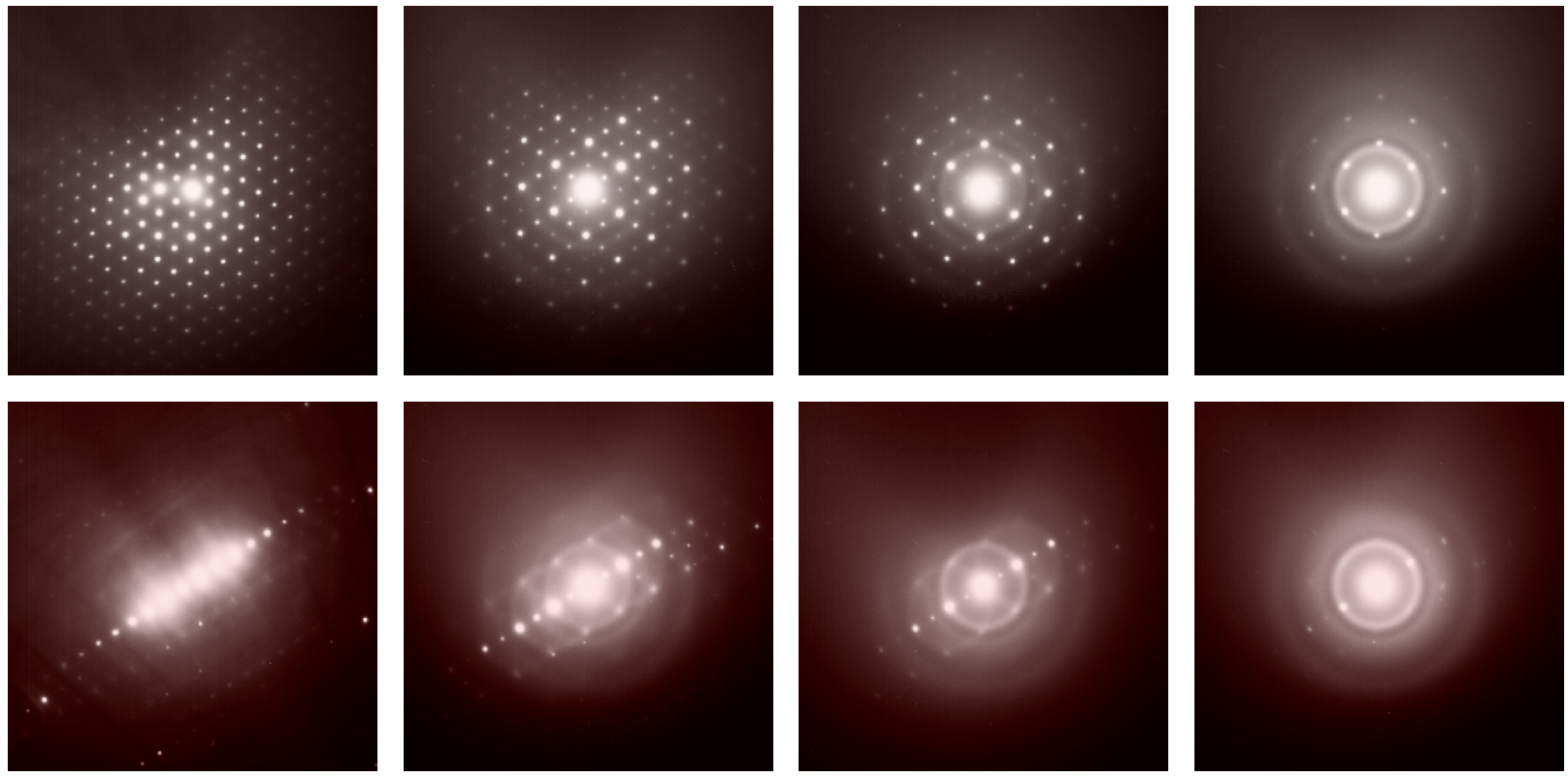

Fig. 2. SAED patterns of unirradiated and irradiated $\mathrm{Gd}_{2} \mathrm{Ti}_{2} \mathrm{O}_{7}$ oriented in the [110] zone axis (top row) and in the $\langle 111\rangle$ reciprocal lattice row (bottom row). The relative dose levels are $0.0 \mathrm{D}_{\mathrm{c}}, 0.2 \mathrm{D}_{\mathrm{c}}$, $0.4 \mathrm{D}_{\mathrm{c}}$, and $0.6 \mathrm{D}_{\mathrm{c}}$ from left to right, where $\mathrm{D}_{\mathrm{c}}$ is the critical dose for amorphization. 